Section Editor Mitchell S.V. Elkind, MD, MS

Yanan Zhang, MD

Hongbo Zheng, MD, $\mathrm{PhD}$

Muke Zhou, MD, PhD Li He, MD, PhD

Correspondence to Dr. He: heli2003new@126.com

Teaching NeuroImages:

\title{
Carotid-cavernous fistula caused by fibromuscular dysplasia
}

A 26-year-old woman developed headache, diplopia, and pulsatile tinnitus in 10 days. Neurologic examination revealed limitation of left eye movement, left dilated pupil with optic disc swelling and mild decreased vision, and a bruit over the left superior orbit. Her family history, laboratory data, and cerebral MRI showed normal findings. Cerebral CT angiography suggested left carotid-cavernous fistula (CCF). Follow-up angiography confirmed left CCF (figure 1). Nevertheless, multiple stenoses interspersed with aneurysmal dilation segments of carotid, vertebral, and renal arteries were found (figure 2). A diagnosis of fibromuscular dysplasia
(FMD) was finally made. FMD is a rare cause of CCF. ${ }^{1,2}$

\section{AUTHOR CONTRIBUTIONS}

Yanan Zhang: drafting/revising the manuscript, including medical writing for content, study concept and design, analysis and interpretation of data. Hongbo Zheng: drafting the manuscript, analysis and interpretation of radiologic data. Muke Zhou: drafting the manuscript, analysis and interpretation of radiologic data. Li He: study supervision.

\section{REFERENCES}

1. Ellis JA, Goldstein H, Connolly ES Jr, Meyers PM. Carotid-cavernous fistulas. Neurosurg Focus 2012;32:E9.

2. Poloskey SL, Olin JW, Mace P, Gornik HL. Fibromuscular dysplasia. Circulation 2012;125:e636-e639.
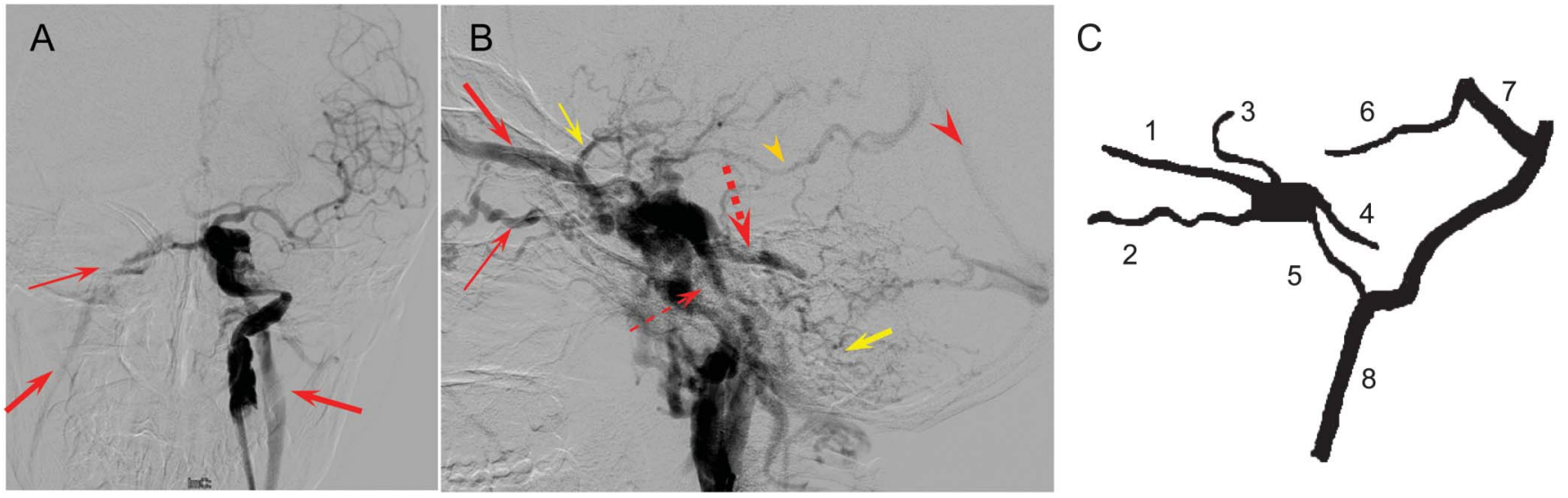

Left internal carotid artery angiography (arterial phase): (A) anterioposterior, (B) lateral view. (A) High-flow carotid-cavernous fistula (CCF) with venous drains to both cavernous sinuses and then to internal jugular veins (thick arrow) via inferior petrosal sinuses (arrow). (B) CCF drains to an enlarged superior ophthalmic vein (thick arrow), to inferior ophthalmic vein (arrow), to superior (thick dotted arrow) and inferior (dotted arrow) petrosal sinus, to straight sinus (large arrowhead) via basal vein of Rosenthal (arrowhead), to sphenoparietal sinus (short arrow), and to some cortical veins (short thick arrow). (C) Schematic drawings of venous drainage: 1 indicates superior ophthalmic vein; 2 , inferior ophthalmic vein; 3 , sphenoparietal sinus; 4, superior petrosal sinus; 5 , inferior petrosal sinus; 6 , basal vein of Rosenthal; 7, straight sinus; 8 , internal jugular veins. 
Figure 2 Angiography

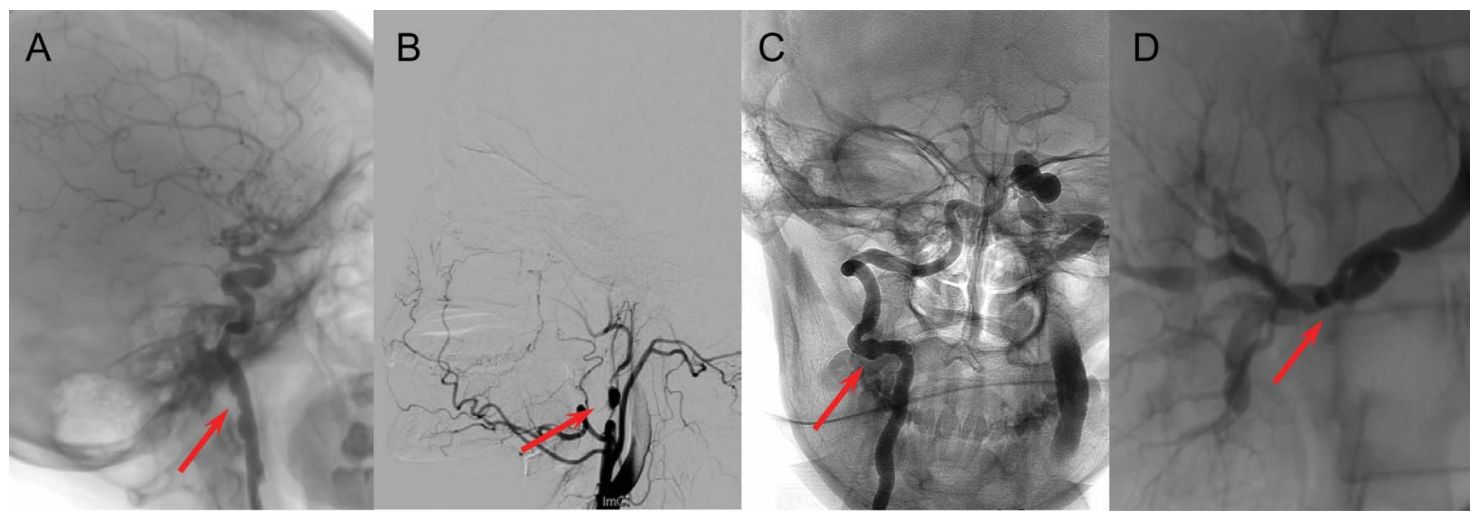

Multiple stenoses interspersed with aneurysmal dilation segments. (A) Right internal carotid artery, (B) left external carotid artery, (C) right vertebral artery, (D) right renal artery. 


\section{Neurology}

Teaching NeuroImages: Carotid-cavernous fistula caused by fibromuscular dysplasia Yanan Zhang, Hongbo Zheng, Muke Zhou, et al.

Neurology 2014;82; $134-\mathrm{e} 135$

DOI 10.1212/WNL.0000000000000319

This information is current as of April 14, 2014

Updated Information \&
Services
Supplementary Material
References
Subspecialty Collections
Permissions \& Licensing
Reprints

Updated Information \&

Supplementary Material

References

Subspecialty Collections

Reprints including high resolution figures, can be found at: http://n.neurology.org/content/82/15/e134.full

Supplementary material can be found at: http://n.neurology.org/content/suppl/2014/04/13/82.15.e134.DC1

This article cites 2 articles, 1 of which you can access for free at: http://n.neurology.org/content/82/15/e134.full\#ref-list-1

This article, along with others on similar topics, appears in the following collection(s):

Diplopia (double vision)

http://n.neurology.org/cgi/collection/diplopia_double_vision Other cerebrovascular disease/ Stroke

http://n.neurology.org/cgi/collection/other_cerebrovascular_disease_s troke

Information about reproducing this article in parts (figures,tables) or in its entirety can be found online at:

http://www.neurology.org/about/about_the_journal\#permissions

Information about ordering reprints can be found online:

http://n.neurology.org/subscribers/advertise

Neurology $®$ is the official journal of the American Academy of Neurology. Published continuously since 1951, it is now a weekly with 48 issues per year. Copyright @ 2014 American Academy of Neurology. All rights reserved. Print ISSN: 0028-3878. Online ISSN: 1526-632X.

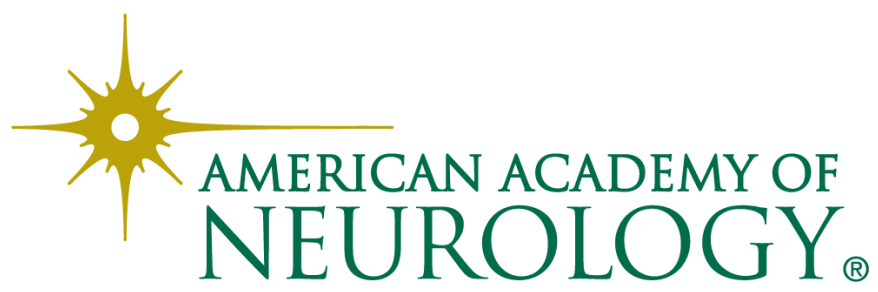

